\title{
Coprescription of Chinese herbal medicine and Western medication among female patients with breast cancer in Taiwan: analysis of national insurance claims
}

This article was published in the following Dove Press journal:

Patient Preference and Adherence

7 May 2014

Number of times this article has been viewed

\begin{abstract}
Bih-Ru Wang ${ }^{1,2, *}$
Yuh-Lih Chang ${ }^{1,2, *}$

Tzeng-Ji Chen ${ }^{3,4}$

Jen-Hwey Chiu ${ }^{5,6}$

Jing Chong $\mathrm{Wu}^{7}$

Min-Shan $\mathrm{Wu}^{\prime}$

Chia-Lin Chou'

Yueh-Ching Chou ${ }^{1,2,8}$

'Department of Pharmacy, Taipei Veterans General Hospital, Taipei, Taiwan; ${ }^{2}$ Department and Institute of Pharmacology, National Yang-Ming University, Taipei, Taiwan; ${ }^{3}$ Department of Family Medicine, Taipei Veterans General Hospital, Taipei, Taiwan; ${ }^{4}$ Institute of Hospital and Health Care Administration, National Yang-Ming University, Taipei, Taiwan; ${ }^{5}$ Division of General Surgery, Department of Surgery, Taipei Veterans General Hospital, Taipei, Taiwan; 'Institute of Traditional Medicine, School of Medicine, National Yang-Ming University, Taipei, Taiwan; ${ }^{7}$ Traditional Medicine Center, Kaohsiung Veterans General Hospital, Kaohsiung, Taiwan; ${ }^{8}$ College of Pharmacy, Taipei Medical University, Taipei, Taiwan
\end{abstract}

*These authors contributed equally to this work

Correspondence:Yueh-Ching Chou Department of Pharmacy, Taipei Veterans General Hospital, Number 201, Section 2, Shipai Road, Beitou District, Taipei, I I2, Taiwan

Tel +886228757 694

Fax +886228757 643

Email ycchou@vghtpe.gov.tw
Background: Many female breast cancer (FBC) patients take Chinese herbal medicine (CHM) and Western medication (WM) concurrently in Taiwan. Despite the possibility of interactions between the CHM and WM mentioned in previous studies, the pattern of these coprescriptions in FBC patients remains unclear. Hence, the aim of the present study is to investigate the utilization of coprescriptions of CHM and WM among the FBC patients in Taiwan.

Methods: The study was a cross-sectional survey using the sampled cohort in 2009 obtained from the National Health Insurance Research Database in Taiwan. There were 3,507 FBC patients identified from the registry for catastrophic illness patients. Ambulatory visit records, corresponding prescriptions, and the data of beneficiaries belonging to the $\mathrm{FBC}$ patients were further extracted. A total of 1,086 FBC patients used CHM at least once. CHM and WM prescribed within any overlapping duration were defined as coprescriptions.

Results: There were 868 (80.0\%) patients simultaneously receiving CHM and WM. A total of 4,927 CHM prescriptions and 6,358 WM prescriptions were prescribed concurrently. Among these coprescriptions, the most frequently used CHM was jia-wei-xiao-yao-san (21.2\%), and the most frequently coprescribed WM was acetaminophen (38.9\%), followed by tamoxifen (25.5\%). There were 346 patients using systemic adjuvant therapy and CHM concurrently. The most commonly coprescribed CHM with chemotherapy, endocrine therapy, and trastuzumab was xiang-sha-liu-jun-zi-tang, jia-wei-xiao-yao-san, and zhi-gan-cao-tang, respectively.

Conclusion: The combined use of CHM with WM is prevalent. The main purpose of combining CHM with systemic cancer treatment is to alleviate the treatment-related adverse effects. However, the combination may result in the potential risk of drug-herb interactions. Further clinical studies are needed to evaluate the efficacy and safety of the CHM and WM coprescriptions for FBC patients.

Keywords: drug utilization patterns, complementary and alternative medicine, pharmacoepidemiology

\section{Introduction}

Breast cancer is the most frequent cancer and the chief cause of cancer death among women in Taiwan and worldwide. ${ }^{1,2}$ The mortality rate of breast cancer has declined, due to the popularly used screening mammography and the greater use of adjuvant therapies in recent decades. ${ }^{3}$ Modern systemic adjuvant treatments, including cytotoxic chemotherapy, endocrine therapy, and anti-human epidermal growth factor receptor 2 (anti-HER2) therapy, should be selected based on the tumor size, grade, hormone-receptor content, and HER2 status. ${ }^{4-7}$ However, treatment-related adverse effects, such as fatigue, nausea, 
vomiting, hot flashes and other menopausal symptoms, cardiac toxicity, etc, make patients unable to tolerate treatment and lead them to seek additional help. ${ }^{8}$

Many breast cancer patients use complementary and alternative medicine for cancer treatment, immune system enhancement, and symptom relief. ${ }^{9,10}$ Traditional Chinese medicine (TCM), including Chinese herbal medicine (CHM) and acupuncture, is the most popular complementary and alternative medicine modality and plays an important role in the Chinese population. ${ }^{11-13}$ A previous study, conducted in Shanghai, the People's Republic of China, showed that $76.8 \%$ of breast cancer patients used CHM therapy after a diagnosis of breast cancer. ${ }^{9}$ In Taiwan, Lai et al also found that $81.5 \%$ of female breast cancer (FBC) patients used TCM services and received $\mathrm{CHM}$ in $76.8 \%$ of visits during the 10 -year study period. ${ }^{14}$ In the survey conducted by Lin and Chiu, $35.6 \%$ of breast cancer patients used TCM during the 1-year observation period in Taiwan, and CHM (80.5\%) was the most commonly used therapy. ${ }^{15}$ All the studies revealed that the vast majority of the CHM users with breast cancer concurrently used Western medication (WM). This common practice raises concerns about the potential problems of drug-herb interactions.

TCM service is popular in the modern health system covered by the National Health Insurance (NHI) in Taiwan. ${ }^{11,16}$ Despite the possibility of interactions between CHM and WM mentioned in previous reports, the pattern of coprescribed $\mathrm{CHM}$ and WM in breast cancer patients remains unclear. Hence, the aim of the present study is to investigate the utilization of coprescriptions of CHM and WM among patients with breast cancer in Taiwan.

\section{Materials and methods}

\section{Data resources}

The NHI program in Taiwan was implemented in 1995 and has covered approximately $98 \%$ of the total population of Taiwan in recent years (http://nhird.nhri.org.tw/en/index.htm). Both Western medicine and TCM services are covered by NHI. TCM is only reimbursed by NHI for ambulatory care, not for inpatient care. Both claims of Western medicine and TCM visits are required to record diagnoses based on the International Classification of Diseases, Ninth Revision, Clinical Modification (ICD-9-CM) coding system for reimbursement.

All electronic claim data under the NHI Research Database (NHIRD) project are released by the Bureau of NHI (BNHI) for research and are further managed by the National Health Research Institutes (NHRI). ${ }^{17}$ The identification numbers of patients and care providers are deidentified before being sent to the NHRI and are further encrypted before being released to each researcher by the NHRI. Thus, the researchers cannot identify the patients or the care providers. However, the cryptographically scrambled identification numbers remain unique for record linking within the datasets. The present study was exempted from a full review by the Institutional Review Board of the Taipei Veterans General Hospital.

We obtained the database of the complete registry for catastrophic illness patients (RCIP) since 1997 (HV1997HV2009) and the sampled cohort file (Longitudinal Health Insurance Database 2005, LHID2005) in 2009, including the registry for beneficiaries (ID2009), ambulatory visit records (CD2009), and corresponding prescription files (OO2009). The RCIP includes all approved cases of catastrophic illness, including breast cancer. Patients with catastrophic illness should submit the pathology and related laboratory reports for registration with RCIP. The approved cases can waive the copayment for each ambulatory visit and hospitalization.

\section{Study sample}

This was a cross-sectional study. Recruited subjects were FBC patients. Initially, we identified the FBC patients who had ever been registered with a malignant neoplasm of female breast (ICD-9-CM code: 174) from the RCIP. Ambulatory visit records, corresponding prescriptions, and the data of beneficiaries belonging to the FBC patients among the sampled cohort (LHID2005) in 2009 were further extracted by linking with the cryptographic identification numbers.

The FBC patients with at least one TCM visit in 2009 were defined as TCM users. Among TCM users, those who had ever received CHM prescriptions were defined as CHM users. All diagnoses of each CHM visit were considered. We tried to find the most frequently coprescribed CHM and WM and the most commonly coprescribed CHM with systemic adjuvant therapy among FBC patients in 2009. CHM and WM prescribed within any overlapping duration were defined as coprescriptions. Figure 1 illustrates the framework of the CHM users among FBC patients and corresponding prescription selection.

\section{Study drugs}

In Taiwan, CHM generally presents as Chinese herbal products (CHP), which were concentrated herbal extract, or as a traditional form (crude drug slices processed for decoction). However, only CHPs produced by the Good 


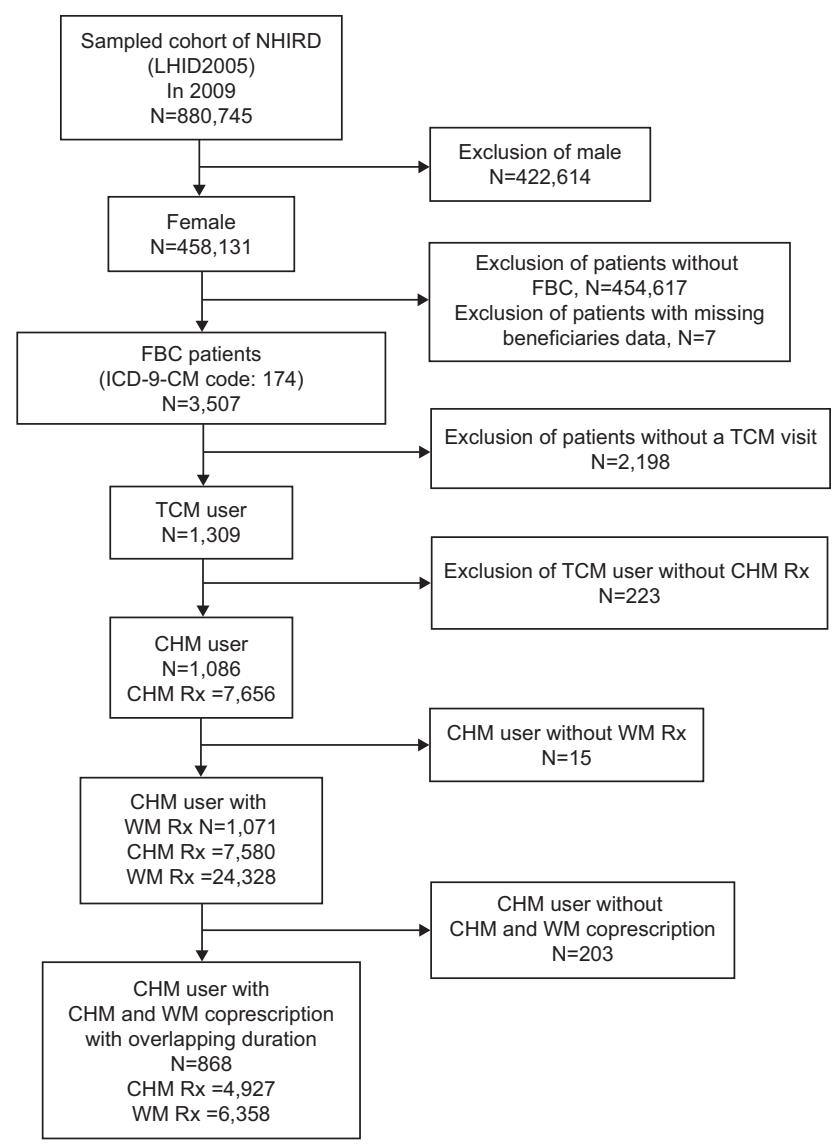

Figure I Framework of $\mathrm{CHM}$ users among $\mathrm{FBC}$ patients and corresponding prescriptions selection.

Abbreviations: NHIRD, National Health Insurance Research Database; LHID, Longitudinal Health Insurance Database; FBC, female breast cancer; TCM, traditional Chinese medicine; CHM, Chinese herbal medicine; Rx, prescription; WM, Western medication; ICD-9-CM, International Classification of Diseases, Ninth Revision, Clinical Modification.

Manufacturing Practice-certified pharmaceutical companies and prescribed by licensed TCM physicians were reimbursed by the NHI. CHPs include single herbs or herbal formulas composed of two or more herbs. We downloaded all the items of the reimbursed CHPs and WM from the BNHI website. ${ }^{18}$ Detailed information of CHPs, including constituents, was obtained from the website of the Committee on Chinese Medicine and Pharmacy. ${ }^{19}$ In addition, WM that was composed of more than one drug was classified by the Anatomical Therapeutic Chemical (ATC) classification system. ${ }^{20}$

Systemic adjuvant agents for breast cancer in the study were specified, based on reimbursement. Chemotherapeutic drugs included cisplatin, carboplatin, cyclophosphamide, fluorouracil, capecitabine, gemcitabine, methotrexate, doxorubicin, epirubicin, mitoxantrone, vinorelbine, vinblastine, docetaxel, paclitaxel, and etoposide. Drugs for endocrine therapies included selective estrogen receptor modulators (SERMs), aromatase inhibitors (AIs), luteinizing hormone-releasing hormone agonists, fluoxymesterone, and megestrol. SERMs included tamoxifen and toremifene. AIs included anastrozole, letrozole, and exemestane. The luteinizing hormone-releasing hormone agonists included goserelin and leuprolide. Trastuzumab was the only included HER2-directed agent.

\section{Data analysis}

Microsoft SQL Server 2008 (Microsoft Corporation, Redmond, WA, USA) was used for data management and computing. The statistical analysis of the data in this study was performed using PASW Statistics for Windows, version 18.0 (SPSS, Inc, Chicago, IL, USA). Descriptive statistics were used for the utilization of CHM and WM coprescriptions in FBC patients.

\section{Results Patient demographics}

A total of 3,507 FBC patients had ambulatory visits in the sampled cohort (LHID2005) in 2009. Of these, 1,309 (37.3\%) were TCM users, and 1,086 (31.0\%) were CHM users. The detailed demographics of the CHM users with FBC are presented in Table 1. Among CHM users with FBC, the highest proportion of patients was $50-59$ years of age (38.7\%). The majority lived in the northern area $(49.2 \%)$ and the highly urbanized towns $(35.6 \%)$ of Taiwan. The most frequent insurance amount range was about 20,000-39,999 New Taiwan Dollars (41.2\%). More than 60\% of CHM users had been diagnosed with breast cancer $<5$ years. About $43.9 \%$ of CHM users were under systemic adjuvant treatment, and $32.6 \%$ of the patients received endocrine therapy alone during 2009. Young patients were more likely to use CHM only.

\section{Diagnoses}

The most common diagnosis for CHM users was "general symptoms" (24.7\%); the second was "malignant neoplasm of female breast" (21.8\%). Approximately one-half of CHM users looked for relieving "symptoms, signs, and ill-defined conditions" $(49.6 \%)$, and the others used CHM to treat respiratory diseases (31.5\%), musculoskeletal diseases (27.7\%), digestive diseases (27.6\%), and neoplasms (23.0\%) (Table 2).

\section{Coprescriptions of CHM and WM}

Among 1,086 FBC patients who were CHM users, 1,071 were under treatment with WM. There were 868 (80.0\%) patients simultaneously receiving CHM and WM. A total of 4,927 CHM prescriptions and 6,358 WM prescriptions were 
Table I Demographics of CHM users among FBC patients in Taiwan, 2009

\begin{tabular}{|c|c|c|c|c|c|c|}
\hline & \multicolumn{2}{|c|}{$\begin{array}{l}\text { Patients with } \mathrm{CHM} \\
\text { and WM coprescription }\end{array}$} & \multicolumn{2}{|c|}{$\begin{array}{l}\text { Patients without } \mathrm{CHM} \\
\text { and WM coprescription }\end{array}$} & \multicolumn{2}{|c|}{ Total } \\
\hline & $\#$ & $\%$ & $\#$ & $\%$ & $\#$ & $\%$ \\
\hline Patient \# & 868 & & 218 & & 1,086 & \\
\hline \multicolumn{7}{|l|}{ Age (years) } \\
\hline$<30$ & 0 & 0.0 & 4 & 1.8 & 4 & 0.4 \\
\hline $30-39$ & 42 & 4.8 & 13 & 6.0 & 55 & 5.1 \\
\hline $40-49$ & 200 & 23.0 & 56 & 25.7 & 256 & 23.6 \\
\hline $50-59$ & 324 & 37.3 & 96 & 44.0 & 420 & 38.7 \\
\hline $60-69$ & 186 & 21.4 & 41 & 18.8 & 227 & 20.9 \\
\hline $70-79$ & 93 & 10.7 & 7 & 3.2 & 100 & 9.2 \\
\hline$\geq 80$ & 23 & 2.6 & 1 & 0.5 & 24 & 2.2 \\
\hline \multicolumn{7}{|l|}{ Diagnostic year for FBC } \\
\hline Before 2004 & 334 & 38.5 & 99 & 45.4 & 433 & 39.9 \\
\hline 2004-2009 & 534 & 61.5 & 119 & 54.6 & 653 & 60.1 \\
\hline \multicolumn{7}{|l|}{ Geographical location } \\
\hline Northern & 421 & 48.5 & 113 & 51.8 & 534 & 49.2 \\
\hline Central & 165 & 19.0 & 45 & 20.6 & 210 & 19.3 \\
\hline Southern & 259 & 29.8 & 58 & 26.6 & 317 & 29.2 \\
\hline Eastern & 23 & 2.6 & 2 & 0.9 & 25 & 2.3 \\
\hline \multicolumn{7}{|l|}{ Urbanization level } \\
\hline I (highest) & 308 & 35.5 & 79 & 36.2 & 387 & 35.6 \\
\hline 2 & 252 & 29.0 & 58 & 26.6 & 310 & 28.5 \\
\hline 3 & 98 & 11.3 & 33 & 15.1 & $13 \mid$ & 12.1 \\
\hline 4 & 106 & 12.2 & 27 & 12.4 & 133 & 12.2 \\
\hline 5 (lowest) & 104 & 12.0 & 21 & 9.6 & 125 & 11.5 \\
\hline \multicolumn{7}{|l|}{ Insurance amount (NTD) } \\
\hline Fixed premium and dependent & 251 & 28.9 & 61 & 28.0 & 312 & 28.7 \\
\hline $1-19,999$ & 156 & 18.0 & 32 & 14.7 & 188 & 17.3 \\
\hline $20,000-39,999$ & 351 & 40.4 & 96 & 44.0 & 447 & 41.2 \\
\hline$\geq 40,000$ & 110 & 12.7 & 29 & 13.3 & 139 & 12.8 \\
\hline \multirow{2}{*}{\multicolumn{7}{|c|}{$\begin{array}{l}\text { Current systemic } \\
\text { adjuvant treatment }{ }^{\mathrm{a}}\end{array}$}} \\
\hline & & & & & & \\
\hline $\mathrm{CT}^{\mathrm{b}}$ & 47 & 5.4 & 10 & 4.6 & 57 & 5.2 \\
\hline $\mathrm{ET}^{\mathrm{c}}$ & 330 & 38.0 & 24 & 11.0 & 354 & 32.6 \\
\hline Trastuzumab & 0 & 0.0 & 0 & 0.0 & 0 & 0.0 \\
\hline $\mathrm{CT}+\mathrm{ET}$ & 45 & 5.2 & 6 & 2.8 & 51 & 4.7 \\
\hline $\mathrm{CT}+$ trastuzumab & 10 & 1.2 & 0 & 0.0 & 10 & 0.9 \\
\hline ET + trastuzumab & 1 & 0.1 & 0 & 0.0 & 1 & 0.1 \\
\hline $\mathrm{CT}+\mathrm{ET}+$ trastuzumab & 4 & 0.5 & 0 & 0.0 & 4 & 0.4 \\
\hline No systemic adjuvant treatment & 431 & 49.7 & 178 & 81.7 & 609 & 56.1 \\
\hline
\end{tabular}

Notes: aPatients have received systemic adjuvant treatment with or without coprescriptions of CHM. 'اncluded cisplatin, carboplatin, cyclophosphamide, fluorouracil, capecitabine, gemcitabine, methotrexate, doxorubicin, epirubicin, mitoxantrone, vinorelbine, vinblastine, docetaxel, paclitaxel, and etoposide. 'Included selective estrogen receptor modulator (tamoxifen, toremifene), aromatase inhibitor (anastrozole, letrozole, exemestane), luteinizing hormone-releasing hormone agonist (goserelin, leuprolide), fluoxymesterone, megestrol.

Abbreviations: CHM, Chinese herbal medicine; FBC, female breast cancer; WM, Western medication; NTD, New Taiwan Dollars; CT, chemotherapy; ET, endocrine therapy.

prescribed concurrently during the study period. Table 3 presents the most commonly used CHM and WM in coprescriptions among FBC patients in Taiwan during 2009. In these coprescriptions, the most frequently used CHM was jia-wei-xiao-yao-san (JWXYS) (21.2\%), and the most frequently coprescribed WM was acetaminophen (38.9\%), followed by tamoxifen (25.5\%).

According to the ATC classification system, analgesics, cough and cold preparations, psycholeptics (including anxiolytics and hypnotics), drugs for acid-related disorders, and endocrine therapy were the most common WM used concurrently with CHM.

There were $346 \mathrm{CHM}$ users receiving systemic adjuvant therapy concurrently. Table 4 shows the most frequently coprescribed CHM with different types of systemic adjuvant therapy. Xiang-sha-liu-jun-zi-tang (27.3\%) and zhi-gan-cao-tang $(40.0 \%)$ were the most commonly used CHM with chemotherapy and anti-HER2 therapy, 
Table 2 Main reasons for CHM use among FBC patients in Taiwan, 2009

\begin{tabular}{|c|c|c|c|}
\hline \multicolumn{2}{|l|}{ Main reasons } & \multirow{2}{*}{$\begin{array}{l}\text { \# patients } \\
(n=I, 086)\end{array}$} & \multirow[t]{2}{*}{$\%$} \\
\hline ICD-9-CM code range ${ }^{a}$ & Disease categories & & \\
\hline 780-799 & Symptoms, signs, ill-defined conditions & 539 & 49.6 \\
\hline $460-519$ & Diseases of respiratory system & 342 & 31.5 \\
\hline $710-739$ & Diseases of musculoskeletal system, connective tissue & 301 & 27.7 \\
\hline $520-579$ & Diseases of digestive system & 300 & 27.6 \\
\hline $140-239$ & Neoplasms & 250 & 23.0 \\
\hline $800-999$ & Injury, poisoning & 140 & 12.9 \\
\hline $580-629$ & Diseases of genitourinary system & 135 & 12.4 \\
\hline $320-389$ & Diseases of nervous system, sense organs & 82 & 7.6 \\
\hline $680-709$ & Diseases of skin, subcutaneous tissue & 73 & 6.7 \\
\hline $390-459$ & Diseases of circulatory system & 65 & 6.0 \\
\hline $240-279$ & Endocrine, nutritional, metabolic diseases, immunity disorders & 40 & 3.7 \\
\hline $290-319$ & Mental disorders & 33 & 3.0 \\
\hline $001-139$ & Infectious, parasitic diseases & 14 & 1.3 \\
\hline $280-289$ & Diseases of blood, blood-forming organs & 9 & 0.8 \\
\hline $740-759$ & Congenital anomalies & 4 & 0.4 \\
\hline $630-679$ & Complications of pregnancy, childbirth, puerperium & 2 & 0.2 \\
\hline
\end{tabular}

Note: aNo patients with the following ICD-9-CM codes were diagnosed: 760-779; V0I-V89; E800-E999.

Abbreviations: CHM, Chinese herbal medicine; FBC, female breast cancer; ICD-9-CM, International Classification of Disease, Ninth Revision, Clinical Modification.

respectively. In addition, JWXYS was the most popular

CHM coprescribed with SERMs or AIs. The ingredients

of CHPs mentioned in the text are shown in Tables S1 and $\mathrm{S} 2$.

\section{Discussion}

To our knowledge, this study is the first population-based pharmacoepidemiology survey of CHM and WM coprescriptions among patients with FBC in Taiwan. Our results

Table 3 Most commonly used CHM and WM in coprescriptions among FBC patients in Taiwan, 2009

\begin{tabular}{|c|c|c|c|c|c|}
\hline \multicolumn{3}{|l|}{ CHM } & \multicolumn{3}{|l|}{ WM } \\
\hline $\begin{array}{l}\text { Chinese herbal products } \\
\text { (Chinese name) }\end{array}$ & $\begin{array}{l}\text { \# patients }(\%) \\
(n=868)\end{array}$ & $\begin{array}{l}\text { \# prescriptions }(\%) \\
(n=4,927)\end{array}$ & Drugs $^{a}$ & $\begin{array}{l}\text { \# patients }(\%) \\
(n=868)\end{array}$ & $\begin{array}{l}\text { \# prescriptions (\%) } \\
(n=6,358)\end{array}$ \\
\hline Jia-wei-xiao-yao-san & $184(2 \mid .2)$ & $495(10.0)$ & Acetaminophen & 338 (38.9) & $706(I I . I)$ \\
\hline Yan-hu-suo & I2I (I3.9) & $230(4.7)$ & Tamoxifen & $221(25.5)$ & $908(14.3)$ \\
\hline Bei-mu & $119(13.7)$ & $284(5.8)$ & Antacids & $217(25.0)$ & $431(6.8)$ \\
\hline Huang-qin & 116 (13.4) & $299(6.1)$ & $\begin{array}{l}\text { Cough suppressants and } \\
\text { expectorants, combinations }\end{array}$ & 199 (22.9) & $404(6.4)$ \\
\hline Dan-shen & $109(12.6)$ & $319(6.5)$ & Diclofenac & $159(18.3)$ & $255(4.0)$ \\
\hline Jie-geng & $108(12.4)$ & $231(4.7)$ & Ambroxol & II5 (I3.2) & |8| (2.8) \\
\hline Suan-zao-ren & 102 (I I.8) & $256(5.2)$ & Intravenous fluids ${ }^{b}$ & $104(12.0)$ & $325(5.1)$ \\
\hline Shao-yao-gan-cao-tang & $100(11.5)$ & $210(4.3)$ & Zolpidem & $104(12.0)$ & $273(4.3)$ \\
\hline Ban-xia-xieh-xin-tang & $98(11.3)$ & $260(5.3)$ & $\begin{array}{l}\text { Nasal decongestants for } \\
\text { systemic use }\end{array}$ & $104(12.0)$ & $196(3.1)$ \\
\hline Shu-jing-huo-xie-tang & $94(10.8)$ & $189(3.8)$ & Dimethicone & $85(9.8)$ & $142(2.2)$ \\
\hline Shou-wu-teng & $93(10.7)$ & $25 I(5.1)$ & Alprazolam & $84(9.7)$ & $229(3.6)$ \\
\hline Huang-qi & $92(10.6)$ & $270(5.5)$ & Lorazepam & $82(9.4)$ & $211(3.3)$ \\
\hline Suan-zao-ren-tang & $91(10.5)$ & $238(4.8)$ & Domperidone & $81(9.3)$ & $148(2.3)$ \\
\hline Ge-gen-tang & $91(10.5)$ & $172(3.5)$ & Amlodipine & $74(8.5)$ & $216(3.4)$ \\
\hline Mai-dong & $90(10.4)$ & $197(4.0)$ & Cimetidine & $73(8.4)$ & $134(2.1)$ \\
\hline Ping-wei-san & $89(10.3)$ & $227(4.6)$ & Mefenamic acid & 7I (8.2) & $120(1.9)$ \\
\hline Chuan-xiong-cha-tiao-san & $88(10.1)$ & $218(4.4)$ & $\begin{array}{l}\text { Corticosteroids, combinations } \\
\text { with antibiotics, topical use }\end{array}$ & $7 \mid(8.2)$ & $110(1.7)$ \\
\hline Yin-qiao-san & $88(10.1)$ & $160(3.2)$ & Ibuprofen & $69(7.9)$ & $110(1.7)$ \\
\hline Ge-gen & $84(9.7)$ & $217(4.4)$ & Chlorzoxazone & $69(7.9)$ & $106(1.7)$ \\
\hline Xuan-shen & $84(9.7)$ & $208(4.2)$ & Dextromethorphan & $69(7.9)$ & $99(1.6)$ \\
\hline
\end{tabular}

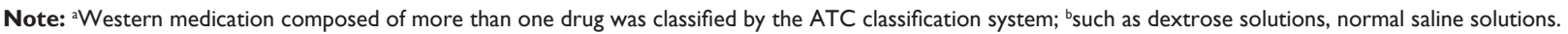
Abbreviations: CHM, Chinese herbal medicine; WM, Western medication; FBC, female breast cancer; ATC, Anatomical Therapeutic Chemical classification system. 
Table 4 Most commonly used CHM coprescribed with systemic adjuvant treatment among FBC patients in Taiwan, 2009

\begin{tabular}{|c|c|c|}
\hline $\begin{array}{l}\text { Chinese herbal products } \\
\text { (Chinese name) }\end{array}$ & $\begin{array}{l}\text { \# patients }(\%) \\
(n=346)\end{array}$ & $\begin{array}{l}\text { \# prescriptions (\%) } \\
(n=2,125)\end{array}$ \\
\hline With chemotherapy & 66 & 357 \\
\hline Xiang-sha-liu-jun-zi-tang & I8 (27.3) & $61(17.1)$ \\
\hline Jia-wei-xiao-yao-san ${ }^{\mathrm{a}, \mathrm{b}}$ & $12(18.2)$ & $24(6.7)$ \\
\hline Huang-qi & II (I6.7) & $32(9.0)$ \\
\hline Dan-shen & II (I6.7) & $27(7.6)$ \\
\hline Pu-gong-ying & II (I6.7) & $26(7.3)$ \\
\hline Ban-xia-xieh-xin-tang & II (I6.7) & $19(5.3)$ \\
\hline Sheng-mai-san & $10(15.2)$ & $29(8.1)$ \\
\hline Mu-dan-pi & $10(15.2)$ & $18(5.0)$ \\
\hline Gan-lu-yinc & $9(13.6)$ & $49(13.7)$ \\
\hline Bai-hua-she-she-cao & $9(13.6)$ & $41(11.5)$ \\
\hline Sha-ren & $9(13.6)$ & $22(6.2)$ \\
\hline Fu-ling & $9(13.6)$ & $21(5.9)$ \\
\hline Bai-zhu & $9(13.6)$ & $18(5.0)$ \\
\hline Suan-zao-ren & $9(13.6)$ & $14(3.9)$ \\
\hline Huang-qin & $9(13.6)$ & $14(3.9)$ \\
\hline With endocrine therapy & 301 & $\mathrm{I}, \mathbf{8 0} \mathrm{I}$ \\
\hline SERM & 221 & 1,320 \\
\hline Jia-wei-xiao-yao-san ${ }^{\mathrm{a}, \mathrm{b}}$ & $5 I(23.1)$ & $177(13.4)$ \\
\hline Suan-zao-ren-tang ${ }^{d}$ & $32(14.5)$ & $103(7.8)$ \\
\hline Ban-xia-xieh-xin-tang & $31(14.0)$ & $55(4.2)$ \\
\hline Suan-zao-ren & $29(13.1)$ & $88(6.7)$ \\
\hline Bei-mu & $29(13.1)$ & $74(5.6)$ \\
\hline Tian-wang-bu-xin-dan ${ }^{a, c}$ & $29(13.1)$ & $73(5.5)$ \\
\hline Yan-hu-suo & $28(12.7)$ & $58(4.4)$ \\
\hline Shou-wu-teng & $27(12.2)$ & $97(7.3)$ \\
\hline Yu-jin & $26(11.8)$ & $97(7.3)$ \\
\hline Huang-qi & $25(11.3)$ & $89(6.7)$ \\
\hline Dan-shen & $25(11.3)$ & $77(5.8)$ \\
\hline Jie-geng & $25(11.3)$ & $52(3.9)$ \\
\hline $\mathrm{Al}$ & 85 & 462 \\
\hline Jia-wei-xiao-yao-san ${ }^{\mathrm{a}, \mathrm{b}}$ & $15(17.6)$ & $32(6.9)$ \\
\hline Huang-qin & $13(15.3)$ & $22(4.8)$ \\
\hline Shao-yao-gan-cao-tang ${ }^{\mathrm{b}}$ & $12(14.1)$ & $23(5.0)$ \\
\hline Jie-geng & $12(14.1)$ & $21(4.5)$ \\
\hline Gan-cao & $11(12.9)$ & $30(6.5)$ \\
\hline Bai-shaob & II (I2.9) & $22(4.8)$ \\
\hline Ban-xia-xieh-xin-tang & $10(11.8)$ & $56(12.1)$ \\
\hline San-qi & $10(11.8)$ & $23(5.0)$ \\
\hline Bei-mu & $10(11.8)$ & $23(5.0)$ \\
\hline Zhi-qiao & $10(11.8)$ & $18(3.9)$ \\
\hline Du-huo-ji-sheng-tang-d & $10(11.8)$ & $17(3.7)$ \\
\hline Huang-lian & $10(11.8)$ & $16(3.5)$ \\
\hline Xu-duan & $10(11.8)$ & $11(2.4)$ \\
\hline Other endocrine therapies & 4 & 27 \\
\hline With anti-HER2 therapy (trastuzumab) & 10 & 95 \\
\hline Zhi-gan-cao-tang & $4(40.0)$ & $13(13.7)$ \\
\hline Gan-lu-yin ${ }^{c}$ & $3(30.0)$ & $21(22.1)$ \\
\hline Sha-ren & $3(30.0)$ & $9(9.5)$ \\
\hline Xiang-sha-liu-jun-zi-tang & $3(30.0)$ & $8(8.4)$ \\
\hline Pu-gong-ying & $3(30.0)$ & $5(5.3)$ \\
\hline Zhen-ren-huo-ming-yin ${ }^{\mathrm{a}}$ & $3(30.0)$ & $5(5.3)$ \\
\hline Tian-hua-fen & $3(30.0)$ & $5(5.3)$ \\
\hline
\end{tabular}

Notes: ${ }^{\mathrm{C} C h i n e s e ~ h e r b a l ~ p r o d u c t s ~ c o n t a i n i n g ~ A n g e l i c a e ~ s i n e n s i s ~ r a d i x . ~}{ }^{\mathrm{b}} \mathrm{Chinese}$ herbal products containing Paeoniae radix alba. ${ }^{\mathrm{c} C h i n e s e}$ herbal products containing Rehmanniae radix praparata. ${ }^{\circ} \mathrm{Chinese}$ herbal products containing Chuanxiong rhizoma.

Abbreviations: CHM, Chinese herbal medicine; FBC, female breast cancer; SERM, selective estrogen receptor modulator; Al, aromatase inhibitor; HER2, human epidermal growth factor receptor 2 . 
highlight the importance of further studies required to evaluate the clinical impact of these coprescriptions for FBC patients.

\section{CHM utilization}

From the present study, the high prevalence $(37.3 \%)$ of TCM usage among FBC patients in Taiwan is in accordance with the survey conducted by Lin and Chiu. ${ }^{15}$ Breast cancer patients are more likely to use TCM than patients with other cancers. ${ }^{21,22} \mathrm{CHM}$ is the dominant type of TCM used by FBC patients and the general population. ${ }^{11,16}$ In our study, $83.0 \%$ $(1,086 / 1,309)$ TCM users received CHM treatment. We also found that 1,071 (98.6\%) CHM users of FBC patients received both CHM and WM during 2009.

Among them, $868 \mathrm{CHM}$ users took CHM and WM concurrently. In the present study, the most common diagnoses for CHM users of FBC patients were "malignant neoplasm of female breast" and "general symptoms", such as nausea, vomiting, fatigue, etc. However, treatment for breast cancer accounted for only $21.8 \%$, which is in accordance with the findings of the previous survey in Taiwan. ${ }^{14,15}$ This is significantly lower than the results from Shanghai, which found that more than $90 \%$ of FBC patients used CHM for fighting cancer. ${ }^{9}$ The different results may also relate to the different methods of data collection used by our study and the study from Shanghai.

Because the study from Shanghai used directparticipant interviews, it may not directly compare to our results. In addition, our study found that diseases of the digestive, respiratory, musculoskeletal, and genitourinary systems were the most common reasons for using CHM. The purposes of CHM usage are consistent with the results of the general population surveys in Taiwan. ${ }^{11,16}$ Obviously, CHM usage among FBC patients in Taiwan may be regarded as an add-on therapy rather than a substitute modality.

\section{Possible reasons for coprescriptions}

Menopausal syndrome is the major complication among breast cancer patients under chemotherapy and endocrine therapy. ${ }^{8}$ Menopausal complications - including hot flashes, night sweats, insomnia, etc - seriously impact the quality of life of breast cancer patients. Therefore, most of them search for management strategies. According to the study conducted in Shanghai, about 29.4\% of breast cancer patients use CHM to lessen menopausal symptoms. Patients with menopausal symptoms or who had used tamoxifen in the past were prone to use $\mathrm{CHM}^{9}{ }^{9}$ In coprescriptions, we found that JWXYS was the most frequently used CHM, and tamoxifen was the most commonly prescribed WM associated with FBC treatment. Furthermore, our study revealed a high coprescription rate between JWXYS and chemotherapy or JWXYS and endocrine therapy. JWXYS, also named dan-zhi-xiao-yao-san, is the principal CHM for the relief of menopausal syndrome. ${ }^{23-26}$ The clinical effects of JWXYS were documented in the classics of traditional medicine and reported by clinical studies. ${ }^{27,28}$ This implies that many FBC patients under modern systemic cancer treatment suffered from menopausal symptoms and sought CHM therapy concurrently. The results of the present study are compatible with the Shanghai survey. ${ }^{9}$ Our results also found that a considerable proportion of CHM was likely to be used for insomnia when combined with SERMs. These CHM include suan-zao-ren-tang, tian-wang-bu-xin-dan, suan-zao-ren (Ziziphi spinosae semen) and shou-wu-teng (Polygoni multiflori caulis), etc. ${ }^{29-34}$

Musculoskeletal problems, such as arthralgia and osteoporosis, that result from AI treatment are significantly more than those caused by tamoxifen treatment. ${ }^{35}$ Joint pain and musculoskeletal pain are the most commonly reported troublesome adverse effects from AI users. ${ }^{36,37}$ Our results found that most CHM coprescribed with AIs was used for arthritis and pain relief. For example, du-huo-ji-sheng-tang and xu-duan (Dipsaci radix) are used to treat osteoarthritis, osteoporosis, and other bone diseases. ${ }^{38-41}$ Shao-yao-gan-caotang is used to relieve dysmenorrhea and muscle pain. ${ }^{42}$

Cardiac toxicity is one of the major concerns for patients with anthracycline-based chemotherapy and trastuzumab treatment. ${ }^{43,44}$ In contrast to anthracycline-related cardiomyopathy and heart failure, cardiotoxicity of trastuzumab is often manifested by asymptomatic left ventricular ejection fraction reduction, palpitations, and - less often - by heart failure. Sheng-mai-san is a famous herbal formula used for the treatment of heart failure and ischemic heart disease. ${ }^{45}$ The present study found that many FBC patients treated with chemotherapy used sheng-mai-san concurrently. On the other hand, the study revealed that zhi-gan-cao-tang was one of the commonly coprescribed CHM with trastuzumab. Zhi-gan-cao-tang is traditionally used for reversing irregular cardiac rhythm. ${ }^{46}$

In the present study, we also found that many CHM coprescribed with chemotherapy were used for relieving gastrointestinal symptoms. The most frequently coprescribed xiang-sha-liu-jun-zi-tang is a famous formula for the treatment of functional dyspepsia. ${ }^{47}$ Ban-xia-xie-xin-tang can mitigate nausea and vomiting caused by systemic cancer 
therapy. ${ }^{48,49}$ The other single herbs such as sha-ren (Amomi fructus) and bai-zhu (Atractylodis macrocephalae rhizoma) can modulate gastrointestinal symptoms. ${ }^{50,51}$

Besides relieving treatment-related symptoms, other commonly coprescribed CHM are also applied to fight cancer. For example, pu-gong-ying (Taraxaci herba), baihua-she-she-cao (Hedyotis diffusae herba), yu-jin (Curcumae radix), and zhen-ren-huo-ming-yin all have shown antitumor effects. ${ }^{52-54}$ Huang-qin (Scutellariae radix) can enhance the antitumor effect. ${ }^{55}$ Huang-qi (Astragali radix) has an anticancer effect and boosts the immune function. ${ }^{56}$ Overall, one of the main purposes of CHM when combined with systemic cancer treatment was to alleviate the treatmentrelated adverse effects.

\section{Possible interactions between CHM and SERM/anti-HER2 therapy}

Recently, there was a small cohort study, and a case report found that si-wu-tang (SWT), one of the popular Chinese herbal formulas for the regulation of menstruation, might have mitogenic potential side effects on breast duct cells in long-term use. ${ }^{57}$

Moreover, SWT and its constituents, Angelicae sinensis radix, Paeoniae radix alba, Chuanxiong rhizome, and Rehmanniae radix preparata, have been proven to stimulate mammary duct cell growth by the activation of estrogen receptor $\alpha$ and HER-2 signaling in cell line studies..$^{58,59}$ Another study demonstrated that, based on in vivo and in vitro studies, SWT respectively reversed tamoxifen- and trastuzumab-induced antiproliferative effects. ${ }^{60}$ Therefore, it is necessary to cautiously evaluate the coprescriptions of CHM and WM for breast cancer patients.

Our study found that some CHM coprescribed with SERM or with trastuzumab contained parts of the constituents of SWT. Examples are: JWXYS contains Angelicae sinensis radix and Paeoniae radix alba; suan-zao-ren-tang contains Chuanxiong rhizoma; and tian-wang-bu-xin-dan contains Angelicae sinensis radix and Rehmanniae radix praparata. It is still unknown whether these formulas containing these single herbs have the same potential risk to reverse tamoxifen- or trastuzumab-induced antiproliferative effects.

Furthermore, the observed results from in vitro or in vivo studies cannot be extrapolated to those in humans. It is better to remind patients to pay more attention when using CHM and tamoxifen or trastuzumab treatment concurrently. Further clinical studies are required to explore the risk of these interactions.

\section{Limitation}

Our study has several potential limitations.

First, breast cancer in situ, including ductal carcinoma in situ and lobular carcinoma in situ, was not included as a catastrophic illness in the Taiwan NHI program. In addition, some CHM services were self-paid and not covered by the NHI program, including CHM services provided by non-NHI-contracted health care institutions, or self-paid traditional-form Chinese herbal remedies, etc. The lack of above data leads to the underestimation of CHM utilization for FBC patients.

Second, due to the lack of information, including cancer stages, lab data, clinical symptoms, and survival data in NHIRD, the study was neither to evaluate the relationship between disease severities and CHM usage nor to elucidate the effects of CHM therapies.

Third, we defined coprescriptions as CHM and WM prescribed within any overlapping duration. However, we cannot verify the concurrent use of CHM and WM.

Finally, the clinical effects of interactions between CHM and SERM or CHM and anti-HER2 therapy cannot be confirmed.

\section{Conclusion}

CHM use in FBC patients is popular in Taiwan. The main purpose of CHM combined with systemic cancer treatment is to alleviate the treatment-related adverse effects. A high prescription rate of CHM combined with WM may lead to possible drug-herb interactions. Further research is needed to evaluate the clinical impact of CHM and WM coprescriptions for FBC patients.

\section{Acknowledgments}

This study is based, in part, on data from the NHIRD provided by the BNHI, Department of Health, and managed by the NHRI. The interpretations and conclusions contained herein do not represent those of the BNHI, Department of Health, or the NHRI. This study was supported by grants from Taipei Veterans General Hospital (V100A-058) in Taiwan. Sponsors had no role in the design, analysis, or presentation of this study.

\section{Disclosure}

The authors report no conflicts of interest in this work.

\section{References}

1. Jemal A, Bray F, Center MM, Ferlay J, Ward E, Forman D. Global cancer statistics. CA Cancer J Clin. 2011;61(2):69-90. 
2. Bureau of Health Promotion, Department of Health. Cancer registry annual report, 2010, Taiwan. 2013. Available from: http://www.hpa. gov.tw/BHPNet/Portal/File/StatisticsFile/201305061037065219/99\% Е5\%B9\%B4\%E7\%99\%8C\%E7\%97\%87\%Е7\%99\%BB\%E8\%A8\%9 8\%E5\%A0\%B1\%E5\%91\%8A.pdf. Accessed April 29, 2014.

3. Berry DA, Cronin KA, Plevritis SK, et al; Cancer Intervention and Surveillance Modeling Network (CISNET) Collaborators. Effect of screening and adjuvant therapy on mortality from breast cancer. $N E n g l$ J Med. 2005;353(17):1784-1792.

4. Early Breast Cancer Trialists' Collaborative Group (EBCTCG). Effects of chemotherapy and hormonal therapy for early breast cancer on recurrence and 15-year survival: an overview of the randomised trials. Lancet. 2005;365(9472):1687-1717.

5. Howell A, Cuzick J, Baum M, et al; ATAC Trialists' Group. Results of the ATAC (Arimidex, Tamoxifen, Alone or in Combination) trial after completion of 5 years' adjuvant treatment for breast cancer. Lancet. 2005;365(9453):60-62.

6. Swain SM, Jeong JH, Geyer CE Jr, et al. Longer therapy, iatrogenic amenorrhea, and survival in early breast cancer. N Engl J Med. 2010; 362(22):2053-2065.

7. Hudis CA. Trastuzumab - mechanism of action and use in clinical practice. N Engl J Med. 2007;357(1):39-51.

8. Shapiro CL, Recht A. Side effects of adjuvant treatment of breast cancer. N Engl J Med. 2001;344(26):1997-2008.

9. Chen Z, Gu K, Zheng Y, Zheng W, Lu W, Shu XO. The use of complementary and alternative medicine among Chinese women with breast cancer. J Altern Complement Med. 2008;14(8):1049-1055.

10. Wanchai A, Armer JM, Stewart BR. Complementary and alternative medicine use among women with breast cancer: a systematic review. Clin J Oncol Nurs. 2010;14(4):E45-E55.

11. Chen FP, Chen TJ, Kung YY, et al. Use frequency of traditional Chinese medicine in Taiwan. BMC Health Serv Res. 2007;7:26.

12. Chung V, Wong E, Woo J, Lo SV, Griffiths S. Use of traditional Chinese medicine in the Hong Kong special administrative region of China J Altern Complement Med. 2007;13(3):361-367.

13. Hesketh T, Zhu WX. Health in China. Traditional Chinese medicine: one country, two systems. BMJ. 1997;315(7100):115-117.

14. Lai JN, Wu CT, Wang JD. Prescription pattern of Chinese herbal products for breast cancer in Taiwan: a population-based study. Evid Based Complement Alternat Med. 2012;2012:891893.

15. Lin YH, Chiu JH. Use of Chinese medicine by women with breast cancer: a nationwide cross-sectional study in Taiwan. Complement Ther Med. 2011;19(3):137-143.

16. Chang LC, Huang N, Chou YJ, Lee CH, Kao FY, Huang YT. Utilization patterns of Chinese medicine and Western medicine under the National Health Insurance Program in Taiwan, a population-based study from 1997 to 2003. BMC Health Serv Res. 2008;8:170.

17. National Health Insurance Research Database [database on the Internet] Taipei, Taiwan: National Health Research Institutes; 2003. Available from: http://nhird.nhri.org.tw/. Accessed January 5, 2012.

18. National Health Insurance Administartion Ministry of Health and Welfare [webpage on the Internet]. [Bureau of National Health Insurance: List of reimbursed drugs]. Available from: http://www.nhi.gov. tw/webdata/webdata.aspx?menu=17\&menu_id=879\&webdata_id=873. Accessed January 5, 2012. Chinese.

19. Department of Chinese Medicine and Pharmacy. [Database of registered Chinese herbal medicines-current and withdrawn]. Available from: http://www.mohw.gov.tw/cht/DOCMAP/DM1.aspx?f_list_no=499. Accessed April 23, 2014. Chinese.

20. World Health Organization Collaborating Centre for Drug Statistics Methodology. Guidelines for ATC classification and DDD assignment 2013. Oslo, Norway: World Health Organization; 2012. Available from: http://www.whocc.no/filearchive/publications/1_2013guidelines.pdf. Accessed January 5, 2012.

21. Lin YH, Chen KK, Chiu JH. Coprescription of Chinese Herbal Medicine and Western Medications among Prostate Cancer Patients: A Population-Based Study in Taiwan. Evid Based Complement Alternat Med. 2012;2012:147015.
22. Lin YH, Chiu JH. Use of Chinese Medicine among patients with liver cancer in Taiwan. J Altern Complement Med. 2010;16(5): $527-528$.

23. Chen HY, Lin YH, Wu JC, et al. Prescription patterns of Chinese herbal products for menopausal syndrome: analysis of a nationwide prescription database. J Ethnopharmacol. 2011;137(3):1261-1266.

24. Yang YH, Chen PC, Wang JD, Lee CH, Lai JN. Prescription pattern of traditional Chinese medicine for climacteric women in Taiwan. Climacteric. 2009;12(6):541-547.

25. Scheid V, Ward T, Cha WS, Watanabe K, Liao X. The treatment of menopausal symptoms by traditional East Asian medicines: review and perspectives. Maturitas. 2010;66(2):111-130.

26. Scheid V, Ward T, Tuffrey V. Comparing TCM textbook descriptions of menopausal syndrome with the lived experience of London women at midlife and the implications for Chinese medicine research. Maturitas. 2010;66(4):408-416.

27. Chen LC, Tsao YT, Yen KY, Chen YF, Chou MH, Lin MF. A pilot study comparing the clinical effects of Jia-Wey Shiau-Yau San, a traditional Chinese herbal prescription, and a continuous combined hormone replacement therapy in postmenopausal women with climacteric symptoms. Maturitas. 2003;44(1):55-62.

28. Lai JN, Hwang JS, Chen HJ, Wang JD. Finished herbal product as an alternative treatment for menopausal symptoms in climacteric women. J Altern Complement Med. 2005;11(6):1075-1084.

29. Chen FP, Jong MS, Chen YC, et al. Prescriptions of Chinese Herbal Medicines for Insomnia in Taiwan during 2002. Evid Based Complement Alternat Med. 2011;2011:236341.

30. Yeh CH, Arnold CK, Chen YH, Lai JN. Suan zao ren tang as an original treatment for sleep difficulty in climacteric women: a prospective clinical observation. Evid Based Complement Alternat Med. 2011;2011:673813.

31. Yeung WF, Chung KF, Poon MM, et al. Prescription of Chinese herbal medicine and selection of acupoints in pattern-based traditional Chinese medicine treatment for insomnia: a systematic review. Evid Based Complement Alternat Med. 2012;2012:902578.

32. Peng WH, Hsieh MT, Lee YS, Lin YC, Liao J. Anxiolytic effect of seed of Ziziphus jujuba in mouse models of anxiety. J Ethnopharmacol. 2000;72(3):435-441.

33. Jiang JG, Huang XJ, Chen J. Separation and purification of saponins from Semen Ziziphus jujuba and their sedative and hypnotic effects. J Pharm Pharmacol. 2007;59(8):1175-1180.

34. Ye R, Yuan ZZ, Dai CX. [Intervention of tianwang buxin decoction combined with dormancy hygiene education for treatment of sub-healthy insomnia patients of yin deficiency fire excess syndrome]. Zhongguo Zhong Xi Yi Jie He Za Zhi. 2011;31(5):618-621. Chinese [with English abstract].

35. Smith IE, Dowsett M. Aromatase inhibitors in breast cancer. $N$ Engl J Med. 2003;348(24):2431-2442.

36. Felson DT, Cummings SR. Aromatase inhibitors and the syndrome of arthralgias with estrogen deprivation. Arthritis Rheum. 2005;52(9):2594-2598.

37. Mao JJ, Chung A, Benton A, et al. Online discussion of drug side effects and discontinuation among breast cancer survivors. Pharmacoepidemiol Drug Saf. 2013;22(3):256-262.

38. Liu M, Xiao GG, Rong P, et al. Therapeutic effects of radix dipsaci, pyrola herb, and Cynomorium songaricum on bone metabolism of ovariectomized rats. BMC Complement Altern Med. 2012; 12:67.

39. Chen CW, Sun J, Li YM, Shen PA, Chen YQ. Action mechanisms of du-huo-ji-sheng-tang on cartilage degradation in a rabbit model of osteoarthritis. Evid Based Complement Alternat Med. 2011;2011:571479.

40. Lai JN, Chen HJ, Chen CC, Lin JH, Hwang JS, Wang JD. Duhuo jisheng tang for treating osteoarthritis of the knee: a prospective clinical observation. Chin Med. 2007;2:4.

41. Shih WT, Yang YH, Chen PC. Prescription patterns of Chinese herbal products for osteoporosis in Taiwan: a population-based study. Evid Based Complement Alternat Med. 2012;2012:752837. 
42. Hinoshita F, Ogura Y, Suzuki Y, et al. Effect of orally administered shao-yao-gan-cao-tang (Shakuyaku-kanzo-to) on muscle cramps in maintenance hemodialysis patients: a preliminary study. Am J Chin Med. 2003;31(3):445-453.

43. Singal PK, Iliskovic N. Doxorubicin-induced cardiomyopathy. NEngl J Med. 1998;339(13):900-905.

44. Perez EA, Rodeheffer R. Clinical cardiac tolerability of trastuzumab. J Clin Oncol. 2004;22(2):322-329.

45. Zheng H, Chen Y, Chen J, Kwong J, Xiong W. Shengmai (a traditional Chinese herbal medicine) for heart failure [review]. Cochrane Database Syst Rev. 2011;2:CD005052.

46. Chen WG, Ba ZM. Prof. ZHANG Yi's experience in treating severe arrhythmia. J Tradit Chin Med. 2010;30(1):47-50.

47. Xiao Y, Liu YY, Yu KQ, Ouyang MZ, Luo R, Zhao XS. Chinese herbal medicine liu jun zi tang and xiang sha liu jun zi tang for functional dyspepsia: meta-analysis of randomized controlled trials. Evid Based Complement Alternat Med. 2012;2012:936459.

48. Xu H, Zhang WX. Modern Applications of Modified Ban Xia Xie Xin Tang and Their Development. Australian Journal of Acupuncture and Chinese Medicine. 2008;3(1):25-30.

49. Zhao L, Zhang S, Wang Z, et al. Efficacy of modified ban xia xie xin decoction on functional dyspepsia of cold and heat in complexity syndrome: a randomized controlled trial. Evid Based Complement Alternat Med. 2013;2013:812143.

50. Yamazaki T, Matsushita Y, Kawashima K, Someya M, Nakajima Y, Kurashige T. Evaluation of the pharmacological activity of extracts from amomi semen on the gastrointestinal tracts. J Ethnopharmacol. 2000;71(1-2):331-335.

51. Bose S, Kim H. Evaluation of In Vitro Anti-Inflammatory Activities and Protective Effect of Fermented Preparations of Rhizoma Atractylodis Macrocephalae on Intestinal Barrier Function against Lipopolysaccharide Insult. Evid Based Complement Alternat Med. 2013;2013:363076
52. Kim SH, Ahn BZ, Ryu SY. Antitumour effects of ursolic acid isolated from Oldenlandia diffusa. Phytother Res. 1998;12(8):553-556.

53. Lu B, Xu L, Yu L, Zhang L. Extract of radix curcumae prevents gastric cancer in rats. Digestion. 2008;77(2):87-91.

54. Lu B, Yu L, Xu L, Chen H, Zhang L, Zeng Y. The effects of radix curcumae extract on expressions of VEGF, COX-2 and PCNA in gastric mucosa of rats fed with MNNG. Curr Pharm Biotechnol. 2010;11(3):313-317.

55. Kumagai T, Müller CI, Desmond JC, Imai Y, Heber D, Koeffler HP. Scutellaria baicalensis, a herbal medicine: anti-proliferative and apoptotic activity against acute lymphocytic leukemia, lymphoma and myeloma cell lines. Leuk Res. 2007;31(4):523-530.

56. Kurashige S, Akuzawa Y, Endo F. Effects of astragali radix extract on carcinogenesis, cytokine production, and cytotoxicity in mice treated with a carcinogen, N-butyl-N'-butanolnitrosoamine. Cancer Invest. 1999;17(1):30-35.

57. Chang CJ, Chiu JH, Wu CW, Lui WY. Herb-related aneuploidy in breast fibroadenoma. BMJ Case Rep. Epub 2009 May 12.

58. Chang CJ, Chiu JH, Tseng LM, et al. Si-Wu-Tang and its constituents promote mammary duct cell proliferation by up-regulation of HER-2 signaling. Menopause. 2006;13(6):967-976.

59. Chang CJ, Chiu JH, Tseng LM, et al. Modulation of HER2 expression by ferulic acid on human breast cancer MCF7 cells. Eur J Clin Invest. 2006;36(8):588-596.

60. Chen JL, Wang JY, Tsai YF, et al. In vivo and in vitro demonstration of herb-drug interference in human breast cancer cells treated with tamoxifen and trastuzumab. Menopause. 2013;20(6):646-654. 


\section{Supplementary materials}

Table SI Chinese herbal products

\begin{tabular}{|c|c|}
\hline CHPs of single herb (Chinese name) & Ingredient herb (Latin name) \\
\hline Bai-hua-she-she-cao & Hedyotidis diffusae herba \\
\hline Bai-shao & Paeoniae radix alba \\
\hline Bai-zhu & Atractylodis macrocephalae rhizoma \\
\hline Bei-mu & Fritillaria bulbus \\
\hline Dan-shen & Salviae miltiorrhizae radix et rhizoma \\
\hline Fu-ling & Poria \\
\hline Gan-cao & Glycyrrhizae radix et rhizoma \\
\hline Ge-gen & Puerariae lobatae radix \\
\hline Huang-lian & Coptidis rhizoma \\
\hline Huang-qi & Astragali radix \\
\hline Huang-qin & Scutellariae radix \\
\hline Jie-geng & Platycodonis radix \\
\hline Mai-dong & Ophiopogonis radix \\
\hline Mu-dan-pi & Moutan cortex \\
\hline Pu-gong-ying & Taraxaci herba \\
\hline San-qi & Notoginseng radix et rhizoma \\
\hline Sha-ren & Amomi fructus \\
\hline Shou-wu-teng & Polygoni multiflori caulis \\
\hline Suan-zao-ren & Ziziphi spinosae semen \\
\hline Tian-hua-fen & Trichosanthis radix \\
\hline Xuan-shen & Scrophulariae radix \\
\hline Xu-duan & Dipsaci radix \\
\hline Yan-hu-suo & Corydalis rhizoma \\
\hline Yu-jin & Curcumae radix \\
\hline Zhi-qiao & Aurantii fructus \\
\hline
\end{tabular}

Abbreviation: CHPs, Chinese herbal products.

Table S2 Chinese herbal products of herbal formulas

\begin{tabular}{ll}
\hline CHPs of herbal formula (Chinese name) & Ingredient herbs (Latin name) \\
\hline Ban-xia-xieh-xin-tang & Pinelliae rhizoma, Scutellariae radix, Zingiberis rhizoma, Ginseng radix et rhizoma, Coptidis \\
rhizoma, Jujubae fructus, Glycyrrhizae radix et rhizoma preparata cum melle & Angelicae dahuricae radix, Glycyrrhizae radix et rhizoma, Notopterygii rhizoma et radix, \\
Chuan-xiong-cha-tiao-san & Schizonepetae herba, Chuanxiong rhizoma, Asari radix et rhizoma, Saposhnikoviae radix, \\
& Menthae haplocalycis herba \\
Du-huo-ji-sheng-tang & Angelicae pubescentis radix, Taxilli herba, Eucommiae cortex, Achyranthis bidentatae \\
& radix, Asari radix et rhizoma, Gentianae macrophyllae radix, Poria, Cinnamomi cortex, \\
& Saposhnikoviae radix, Chuanxiong rhizoma, Ginseng radix et rhizoma, Glycyrrhizae radix et \\
rhizoma, Angelicae sinensis radix, Paeoniae radix alba, Rehmanniae radix \\
Gan-lu-yin & Rehmanniae radix praparata, Ophiopogonis radix, Aurantii fructus, Glycyrrhizae radix et \\
& rhizoma praparata cum melle, Artemisiae scopariae herba, Eriobotryae folium, Dendrobii \\
Ge-gen-tang & caulis, Scutellariae radix, Rehmanniae radix, Asparagi radix \\
& Puerariae lobatae radix, Ephedrae herba, Cinnamomi ramulus, Paeoniae radix alba, \\
Glycyrrhizae radix et rhizoma praparata cum melle, Zingiberis rhizoma recens, Jujubae & \\
fia-wei-xiao-yao-san & Angelicae sinensis radix, Atractylodis macrocephalae rhizoma, Paeoniae radix alba, Bupleuri \\
radix, Poria, Glycyrrhizae radix et rhizoma praparata cum melle, Moutan cortex, Gardeniae & fructus, Zingiberis rhizoma praeparatum, Menthae haplocalycis herba \\
Ping-wei-san & Citri reticulatae pericarpium, Magnoliae officinalis cortex, Glycyrrhizae radix et rhizoma \\
praparata cum melle, Atractylodis rhizoma, Zingiberis rhizoma recens, Jujubae fructus & Paeoniae radix alba, Glycyrrhizae radix et rhizoma praparata cum melle \\
Shao-yao-gan-cao-tang & Ginseng radix et rhizoma, Ophiopogonis radix, Schisandrae chinensis fructus \\
Suan-zao-ren-tang & Ziziphi spinosae semen, Anemarrhenae rhizoma, Poria, Chuanxiong rhizoma, Glycyrrhizae \\
radix et rhizoma
\end{tabular}


Table S2 (Continued)

\begin{tabular}{|c|c|}
\hline CHPs of herbal formula (Chinese name) & Ingredient herbs (Latin name) \\
\hline Tian-wang-bu-xin-dan & $\begin{array}{l}\text { Rehmanniae radix, Scrophulariae radix, Salviae miltiorrhizae radix et rhizoma, Angelicae } \\
\text { sinensis radix, Schisandrae chinensis fructus, Ophiopogonis radix, Polygalae radix, Coptidis } \\
\text { rhizoma, Platycodonis radix, Asparagi radix, Ziziphi spinosae semen, Ginseng radix et } \\
\text { rhizoma, Poria, Platycladi semen, Rehmanniae radix praparata, Acori tatarinowii rhizoma }\end{array}$ \\
\hline Xiang-sha-liu-jun-zi-tang & $\begin{array}{l}\text { Ginseng radix et rhizoma, Atractylodis macrocephalae rhizoma, Poria, Glycyrrhizae radix et } \\
\text { rhizoma, Citri reticulatae pericarpium, Pinelliae rhizoma, Amomi fructus, Aucklandiae radix, } \\
\text { Zingiberis rhizoma recens }\end{array}$ \\
\hline Yin-qiao-san & $\begin{array}{l}\text { Forsythiae fructus, Lonicerae japonicae flos, Platycodonis radix, Menthae haplocalycis } \\
\text { herba, Lophatheri herba, Glycyrrhizae radix et rhizoma, Schizonepetae herba, Sojae semen } \\
\text { praeparatum, Arctii fructus, Phragmitis rhizoma }\end{array}$ \\
\hline Zhen-ren-huo-ming-yin & $\begin{array}{l}\text { Lonicerae japonicae flos, Citri reticulatae pericarpium, Saposhnikoviae radix, Fritillariae } \\
\text { bulbus, Olibanum, Angelicae sinensis radix, Angelicae dahuricae radix, Trichosanthis radix, } \\
\text { Myrrha, Gleditsiae spina, Glycyrrhizae radix et rhizoma }\end{array}$ \\
\hline Zhi-gan-cao-tang & $\begin{array}{l}\text { Glycyrrhizae radix et rhizoma praparata cum melle, Zingiberis rhizoma recens, Cinnamomi } \\
\text { ramulus, Ginseng radix et rhizoma, Rehmanniae radix, Asini corii colla, Ophiopogonis radix, } \\
\text { Cannabis semen, Jujubae fructus }\end{array}$ \\
\hline
\end{tabular}

Abbreviation: CHPs, Chinese herbal products.

\section{Publish your work in this journal}

Patient Preference and Adherence is an international, peer-reviewed, open access journal that focusing on the growing importance of patient preference and adherence throughout the therapeutic continuum. Patient satisfaction, acceptability, quality of life, compliance, persistence and their role in developing new therapeutic modalities and compounds to optimize clinical outcomes for existing disease states are major areas of interest for the journal. This journal has been accepted for indexing on PubMed Central. The manuscript management system is completely online and includes a very quick and fair peer-review system, which is all easy to use. Visit http://www dovepress.com/testimonials.php to read real quotes from published authors.

Submit your manuscript here: http://www.dovepress.com/patient-preference-and-adherence-journal 